\title{
Studies on Postharvest Changes in Physico-Biochemical Properties of Guava (Psidium guajava L.) cv. Sardar Influenced by Different Composite Coatings
}

\author{
Karishma Kohli*, Shailesh Tripathi, Ankit Kumar, Ajit Kumar, \\ Omveer Singh, Rajesh Kumar and Satish Chand
}

Department of Horticulture, Govind Ballabh Pant University of Agriculture and Technology, Pantnagar - 263145, India

*Corresponding author

Keywords

Guava, Composite coatings,

Postharvest quality,

Sardar

Article Info

Accepted:

12 July 2019

Available Online:

10 August 2019

\section{A B S T R A C T}

The main aim of present study was to evaluate the efficacy of different composite edible coatings comprising olive oil, Aloe vera and sodium alginate as base coatings with antioxidant (ascorbic acid $0.1 \%$ ) and calcium gluconate $(0.5 \%)$ as texture enhancer on the postharvest quality attributes of guava cv. 'Sardar' during winter seasons of 2017 and 2018. After treatment, fruits were kept at ambient temperature of $27-29^{\circ} \mathrm{C}$ till 15 days and analyzed for various physico-biochemical parameters while the uncoated fruits serve as control. In all treatments, olive oil composite coatings were effective in maintaining physiological loss in weight $(7.82 \%)$, fruit firmness $\left(1.14 \mathrm{~kg} / \mathrm{cm}^{2}\right)$, TSS $\left(12.52{ }^{0}\right.$ Brix $)$, titratable acidity $(0.24 \%)$, ascorbic acid (267.56 mg/100 g) and chlorophyll content (3.65 $\mathrm{mg} / \mathrm{g}$ ). Hence, it was concluded that coating treatment of olive oil and Aloe vera composite coating can be used for enhancing the shelf life and maintaining postharvest quality of guava cv. Sardar.

\section{Introduction}

Guava (Psidium guajava L.) is an important subtropical climacteric fruit tree native to Southern Mexico and Central America and belongs to family Myrtaceae. It is termed as a common man's fruit and "Apple of the Tropics" as well. Guava fruit is consumed at both green and ripe stage. Moreover, guava fruits are processed commercially into jelly, jam, puree, juice, powder and nectar. Guava fruit is highly perishable and loss of vitamin $\mathrm{C}$ after harvest takes place very fast which reduces its nutritive value unless appropriate postharvest measures are taken (Pal et al., 2004). Guava being a climacteric fruit ripens rapidly after the harvest therefore has short shelf life. Once it is fully ripe, the fruit becomes soggy and consumer preference and marketing quality deteriorate rapidly. Olive oil 
coating extended the shelf life of guava cv. Allahabad Safeda with minimum physiological weight loss (Kamboj and Kaur, 2018). The sodium alginate edible coating on the pear fruits reduced increase in the TSS value during the storage when compared to that of control fruits by slowing down the polysaccharide degradation (Kessiane et al., 2012). Othman et al., 2017 found that sodium alginate based edible films enhanced the physico-chemical quality of coated guava fruits contained essential oil. Ergun and Satici, 2012 found that the green-coloured 'Granny Smith' and red-coloured 'Red Chief' apples treated with Aloe vera gel had minimum weight loss during the storage period. So many studies have been conducted by researchers to enhance the production but little work has been conducted to reduce postharvest losses of guava fruits. However, it is important to reduce the postharvest losses of guava fruits after harvesting. Therefore, the present study was undertaken to determine the effectiveness of postharvest application of composite coatings like Aloe vera gel, olive oil and sodium alginate with food additives (Texture enhancer and antioxidant) for maintaining the quality and enhancing the shelf life of guava fruits.

\section{Materials and Methods}

The experiment was conducted at Postharvest Laboratory, Department of Horticulture, College of Agriculture, Govind Ballabh Pant University of Agriculture and Technology, Pantnagar, U.S. Nagar (Uttarakhand) during two winter seasons of 2017 and 2018. The guava fruits of winter season crops were harvested at $80 \%$ maturity (colour break stage) and collected in plastic crates covered with a thick layer of guava leaves and brought to the laboratory. Fruits with uniform size and colour were selected whereas blemished and diseased fruit were discarded. Prior to the post-harvest treatment, the fruits were washed in potable water. The fruits were then allowed to dry in shade. Composite coatings were prepared by taking the distilled water in a beaker and calculation of each chemical needed for one litre solvent was done except olive oil coatings and accordingly, coatings were prepared for dip treatment of guava. The composite coatings were prepared from base coatings of olive oil, Aloe vera gel and sodium alginate with composite coating materials viz.., calcium gluconate as a texture enhancer (TE) and ascorbic acid as an antioxidant (AO) of the required concentrations for each treatment. Twenty fruits were dipped in each solution for 10 minutes and then air dried except olive oil. In case of olive oil coating, fruits were coated 3-4 times with the help of brush and left for air drying.

The trial was carried out in three replicates. The detail of the treatments include, $\mathrm{T}_{1}$ : Olive oil $(100 \%)+\mathrm{TE}(0.5 \%) ; \mathrm{T}_{2}$ : Olive oil $(100 \%)+\mathrm{TE}(0.5 \%)+\mathrm{AO}(0.1 \%), \mathrm{T}_{3}:$ Aloe vera $(1: 1)+\mathrm{TE}(0.5 \%), \mathrm{T}_{4}$ : Aloe vera $(1: 1)+$ $\mathrm{TE}(0.5 \%)+\mathrm{AO}(0.1 \%), \mathrm{T}_{5}$ : Sodium alginate $(2 \%)+\mathrm{TE}(0.5 \%), \mathrm{T}_{6}$ : Sodium alginate $(2 \%)+$ $\mathrm{TE}(0.5 \%)+\mathrm{AO}(0.1 \%)$, and $\mathrm{T}_{7}$ : the untreated i.e., control. The treated fruits were kept at ambient temperature. The observations on physical and quality parameters were recorded at an interval of 3 days. Physiological loss in weight was recorded by weighing the sample on electronic balance and expressed in grams (g) from which the physiological loss in weight (PLW) was calculated. Fruit firmness was measured using penetrometer and expressed as $\mathrm{kg} / \mathrm{cm}^{2}$. The quality parameter, TSS of the fruit juice was recorded at room temperature using hand refractometer whereas, the parameters like titratable acidity and ascorbic acid were estimated according to the standard method described by Ranganna (1986) while chlorophyll estimation as per method described by Nayek et al., (2014). The data was statistically analyzed in factorial completely randomized design (FCRD) for analysis of variance. 


\section{Results and Discussion}

The data on effect of various treatments and storage periods on physiological loss in weight of guava under ambient condition are presented in Table 1. It clearly showed that all the treatments exerted a significant influence on physiological loss in weight. Minimum physiological loss in weight (9.35 and 6.29\%) was recorded in $\mathrm{T}_{1}$ followed by $\mathrm{T}_{2}(9.48$ and $6.31 \%$ ) while maximum physiological loss in weight observed in control $\mathrm{T}_{7}(19.44 \%$ and $17.30 \%$ ) during both winter seasons of 2017 and 2018 respectively. In pooled data analysis, minimum physiological loss in weight $(7.82 \%)$ was recorded in $\left(\mathrm{T}_{1}\right)$ followed by $7.89 \%$ in $\mathrm{T}_{2}$ and maximum weight loss $(18.37 \%)$ was recorded in control $\left(\mathrm{T}_{7}\right)$. Storage days affected physiological loss in weight significantly which increased gradually irrespective of the treatment as the storage period progressed. Similar observations have been reported in guava by Gad and Zagzog, 2017, Kumar et al., (2017) in guava, who observed that coated fruits had minimum physiological loss in weight during storage period. Loss of weight in fresh fruits is mainly due to the loss of water caused by transpiration and respiration processes (Zhu et al., 2008).

The data regarding influence of composite coatings during storage period on fruit firmness of guava cv. Sardar are mentioned in Table 1. Data presented in Table 1 showed that maximum fruit firmness $\left(0.77 \mathrm{~kg} / \mathrm{cm}^{2}\right.$ and $1.52 \mathrm{~kg} / \mathrm{cm}^{2}$ ) was found in $\mathrm{T}_{1}$ (olive oil $100 \%$ + TE $0.5 \%$ ) in year 2017 and 2018 respectively while minimum fruit firmness was noticed in i.e., control $\mathrm{T}_{7}\left(0.57 \mathrm{~kg} / \mathrm{cm}^{2}\right.$ and $0.95 \mathrm{~kg} / \mathrm{cm}^{2}$ ) during winter seasons of 2017 and 2018, respectively.

Among different coating treatments, pooled analysis data showed maximum fruit firmness $\left(1.14 \mathrm{~kg} / \mathrm{cm}^{2}\right)$ was recorded in $\mathrm{T}_{1}$ followed by
$\mathrm{T}_{2}\left(1.09 \mathrm{~kg} / \mathrm{cm}^{2}\right)$ and minimum $\left(0.76 \mathrm{~kg} / \mathrm{cm}^{2}\right)$ was recorded in control $\left(\mathrm{T}_{7}\right)$. Similar findings were reported by Gad and Zagzog (2017) in guava and Moradinezhad et al., (2018) in ber who also found that fruits coated with edible coatings retained higher firmness as compared to uncoated fruits during storage period.

It was observed that the firmness of fruits reduced as ripening progressed. Similar observations have been reported in pear fruits as loss in firmness during storage occur due to break down of enzymes, loss of water and degradation of pectic substances present in the fruit (Nath et al., 2012). The data on effect of composite coatings and storage periods and their interactions on fruit total soluble solids (TSS) of guava fruits under ambient condition are presented in Table 2. Maximum TSS was recorded (12.57 ${ }^{\circ}$ Brix) in $\mathrm{T}_{2}$ and minimum (6.35 ${ }^{\circ}$ Brix) was recorded in control $\left(\mathrm{T}_{7}\right)$ on $15^{\text {th }}$ day of storage during winter season of 2017.

Table 2 showed that maximum fruit TSS (13.62 ${ }^{\circ}$ Brix) was recorded in $\left(\mathrm{T}_{2}\right)$ and minimum $\left(6.28^{\circ}\right.$ Brix $)$ was recorded in control $\left(\mathrm{T}_{7}\right)$ on $15^{\text {th }}$ day of storage period during winter season of 2018. In pooled analysis (Table 2) maximum fruit TSS (13.10 ${ }^{\circ}$ Brix) was found in $\mathrm{T}_{2}$ (Olive oil $100 \%+\mathrm{TE} 0.5 \%$ +AO $0.1 \%)$ and minimum $\left(6.32{ }^{\circ}\right.$ Brix $)$ was recorded in control $\left(\mathrm{T}_{7}\right)$ on $15^{\text {th }}$ day of storage period. The above results might be attributed to the reason that coating of olive oil retarded the ripening and senescence processes and simultaneously reduced the conversion of starch into sugars. Kamboj and Kaur (2018) reported that olive oil coated fruits retained significantly higher TSS in guava fruits.

The data pertaining to effect of composite coatings and storage periods on fruit titratable acidity of guava under ambient condition are presented in Table 2. 
Table.1 Effect of different composite coatings on physiological loss in weight and fruit firmness (\%) of guava cv. Sardar

\begin{tabular}{|c|c|c|c|c|c|c|c|c|c|c|c|c|c|c|c|c|c|c|c|c|c|}
\hline & \multicolumn{6}{|c|}{ Physiological loss in weight (\%) } & & \multicolumn{7}{|c|}{ Physiological loss in weight (\%) } & \multicolumn{6}{|c|}{ Physiological loss in weight (\%) } & \multirow{3}{*}{$\begin{array}{l}\text { Pooled } \\
\text { Mean }\end{array}$} \\
\hline Year & \multicolumn{6}{|c|}{2017} & Mean & \multicolumn{6}{|c|}{2018} & Mean & \multicolumn{6}{|c|}{ Pooled data } & \\
\hline \multirow{2}{*}{ Treatments } & \multicolumn{6}{|c|}{ Storage intervals (days) } & & \multicolumn{6}{|c|}{ Storage intervals (days) } & & \multicolumn{6}{|c|}{ Storage intervals (days) } & \\
\hline & 0 & 3 & 6 & 9 & 12 & 15 & & 0 & 3 & 6 & 9 & 12 & 15 & & 0 & 3 & 6 & 9 & 12 & 15 & \\
\hline$T_{1}$ & 0.00 & 3.37 & 7.63 & 12.32 & 14.45 & 18.30 & 9.35 & 0.00 & 1.31 & 4.25 & 5.27 & 10.64 & 16.28 & 6.29 & 0.00 & 2.34 & 5.94 & 8.79 & 12.55 & 17.29 & 7.82 \\
\hline $\mathbf{T}_{2}$ & 0.00 & 2.16 & 7.34 & 11.94 & 15.66 & 19.80 & 9.48 & 0.00 & 0.82 & 2.79 & 4.33 & 12.91 & 16.98 & 6.31 & 0.00 & 1.49 & 5.06 & 8.14 & 14.28 & 18.39 & 7.89 \\
\hline $\mathbf{T}_{3}$ & 0.00 & 4.09 & 11.64 & 14.94 & 20.99 & 31.65 & 13.88 & 0.00 & 2.22 & 5.44 & 7.63 & 16.01 & 18.81 & 8.35 & 0.00 & 3.15 & 8.54 & 11.29 & 18.50 & 25.23 & 11.12 \\
\hline $\mathbf{T}_{4}$ & 0.00 & 4.73 & 11.07 & 15.17 & 19.78 & 28.61 & 13.23 & 0.00 & 0.25 & 4.71 & 8.11 & 17.34 & 18.19 & 8.10 & 0.00 & 2.49 & 7.89 & 11.64 & 18.56 & 23.40 & 10.66 \\
\hline $\mathbf{T}_{5}$ & 0.00 & 4.51 & 10.34 & 13.67 & 17.88 & 26.04 & 12.07 & 0.00 & 3.31 & 4.78 & 10.44 & 16.48 & 17.92 & 8.82 & 0.00 & 3.91 & 7.56 & 12.05 & 17.18 & 21.98 & 10.45 \\
\hline$T_{6}$ & 0.00 & 3.68 & 7.77 & 13.86 & 13.08 & 22.75 & 10.19 & 0.00 & 3.12 & 6.43 & 8.29 & 15.71 & 16.45 & 8.33 & 0.00 & 3.40 & 7.10 & 11.07 & 14.39 & 19.60 & 9.26 \\
\hline $\mathbf{T}_{7}$ & 0.00 & 6.00 & 13.82 & 22.31 & 33.53 & 40.96 & 19.44 & 0.00 & 1.79 & 9.94 & 19.11 & 32.81 & 40.13 & 17.30 & 0.00 & 3.89 & 11.88 & 20.71 & 33.17 & 40.54 & 18.37 \\
\hline Mean & 0.00 & 4.07 & 9.94 & 14.88 & 19.33 & 26.87 & & 0.00 & 1.83 & 5.47 & 9.02 & 17.41 & 20.8 & & 0.00 & 2.95 & 7.71 & 11.96 & 18.38 & 23.78 & \\
\hline
\end{tabular}

\begin{tabular}{|c|c|c|c|c|c|c|c|c|}
\hline Factors & $\mathrm{CD}$ at $5 \%$ & $\mathrm{SE}(\mathrm{m})$ & Factors & $\mathrm{CD}$ at $5 \%$ & $\mathrm{SE}(\mathrm{m})$ & Factors & $\mathrm{CD}$ at $5 \%$ & $\mathrm{SE}(\mathrm{m})$ \\
\hline Storage Intervals (S) & 0.184 & 0.066 & Storage Intervals (S) & 0.122 & 0.044 & Storage Intervals (S) & 0.095 & 0.034 \\
\hline Treatments (T) & 0.301 & 0.108 & Treatments $(\mathrm{T})$ & 0.199 & 0.071 & Treatments $(\mathrm{T})$ & 0.155 & 0.056 \\
\hline Interaction $(\mathbf{S} \times \mathbf{T})$ & 0.738 & 0.264 & Interaction $(\mathrm{S} \times \mathrm{T})$ & 0.486 & 0.174 & Interaction $(\mathrm{S} \times \mathrm{T})$ & 0.38 & 0.136 \\
\hline
\end{tabular}

\begin{tabular}{|c|c|c|c|c|c|c|c|c|c|c|c|c|c|c|c|c|c|c|c|c|c|}
\hline & \multicolumn{6}{|c|}{ Fruit firmness $\left(\mathrm{kg} / \mathrm{cm}^{2}\right)$} & & \multicolumn{7}{|c|}{ Fruit firmness $\left(\mathrm{kg} / \mathrm{cm}^{2}\right)$} & \multicolumn{6}{|c|}{ Fruit firmness $\left(\mathrm{kg} / \mathrm{cm}^{2}\right)$} & \\
\hline Year & \multicolumn{6}{|c|}{2017} & Mean & \multicolumn{6}{|c|}{2018} & Mean & \multicolumn{6}{|c|}{ Pooled data } & \multirow{2}{*}{$\begin{array}{l}\text { Poolec } \\
\text { Mean }\end{array}$} \\
\hline \multirow[t]{2}{*}{ Treatments } & \multicolumn{6}{|c|}{ Storage intervals (days) } & & \multicolumn{6}{|c|}{ Storage intervals (days) } & & \multicolumn{6}{|c|}{ Storage intervals (days) } & \\
\hline & 0 & 3 & 6 & 9 & 12 & 15 & & 0 & 3 & 6 & 9 & 12 & 15 & & 0 & 3 & 6 & 9 & 12 & 15 & \\
\hline$T_{1}$ & 1.10 & 1.10 & 1.00 & 0.80 & 0.40 & 0.20 & 0.77 & 2.23 & 2.21 & 1.97 & 1.11 & 0.90 & 0.70 & 1.52 & 1.66 & 1.66 & 1.48 & 0.96 & 0.65 & 0.45 & 1.14 \\
\hline $\mathbf{T}_{2}$ & 1.10 & 1.10 & 1.00 & 0.70 & 0.30 & 0.20 & 0.73 & 2.23 & 2.21 & 1.97 & 1.11 & 0.80 & 0.30 & 1.44 & 1.66 & 1.65 & 1.49 & 0.91 & 0.55 & 0.25 & 1.09 \\
\hline $\mathbf{T}_{3}$ & 1.10 & 0.99 & 0.80 & 0.60 & 0.20 & 0.20 & 0.65 & 2.23 & 1.78 & 1.00 & 0.98 & 0.70 & 0.44 & 1.19 & 1.67 & 1.38 & 0.90 & 0.79 & 0.45 & 0.32 & 0.92 \\
\hline $\mathbf{T}_{4}$ & 1.10 & 0.99 & 0.80 & 0.70 & 0.30 & 0.10 & 0.67 & 2.23 & 1.98 & 0.99 & 0.89 & 0.71 & 0.48 & 1.21 & 1.67 & 1.49 & 0.90 & 0.79 & 0.50 & 0.29 & 0.94 \\
\hline$T_{5}$ & 1.10 & 1.10 & 1.00 & 0.80 & 0.20 & 0.12 & 0.72 & 2.23 & 1.67 & 1.10 & 1.11 & 0.88 & 0.61 & 1.27 & 1.66 & 1.38 & 1.05 & 0.95 & 0.54 & 0.37 & 0.99 \\
\hline$T_{6}$ & 1.10 & 0.99 & 1.00 & 0.60 & 0.20 & 0.10 & 0.67 & 2.23 & 1.77 & 0.99 & 1.12 & 0.67 & 0.61 & 1.23 & 1.67 & 1.38 & 0.99 & 0.86 & 0.43 & 0.35 & 0.95 \\
\hline $\mathbf{T}_{7}$ & 1.10 & 0.99 & 0.60 & 0.40 & 0.20 & 0.10 & 0.57 & 2.23 & 1.19 & 0.99 & 0.57 & 0.38 & 0.32 & 0.95 & 1.66 & 1.09 & 0.80 & 0.49 & 0.29 & 0.21 & 0.76 \\
\hline Mean & 1.10 & 1.04 & 0.89 & 0.66 & 0.26 & 0.15 & & 2.23 & 1.83 & 1.29 & 0.98 & 0.72 & 0.49 & & 1.66 & 1.43 & 1.09 & 0.82 & 0.49 & 0.32 & \\
\hline \multicolumn{3}{|c|}{ Factors } & \multicolumn{2}{|c|}{$\mathrm{CD}$ at $5 \%$} & \multicolumn{3}{|c|}{$\mathrm{SE}(\mathrm{m})$} & \multicolumn{3}{|c|}{ Factors } & \multicolumn{2}{|c|}{$\mathrm{CD}$ at $5 \%$} & \multicolumn{2}{|c|}{$\mathrm{SE}(\mathrm{m})$} & \multicolumn{3}{|c|}{ Factors } & \multicolumn{2}{|c|}{$\mathrm{CD}$ at $5 \%$} & \multicolumn{2}{|c|}{$\mathrm{SE}(\mathrm{m})$} \\
\hline \multicolumn{3}{|c|}{ Storage Intervals (S) } & \multicolumn{2}{|c|}{0.009} & \multicolumn{3}{|c|}{0.003} & \multicolumn{3}{|c|}{ Storage Intervals (S) } & \multicolumn{2}{|c|}{0.014} & \multicolumn{2}{|c|}{0.005} & \multicolumn{3}{|c|}{ Storage Intervals (S) } & \multicolumn{2}{|c|}{0.009} & \multicolumn{2}{|c|}{0.003} \\
\hline \multirow{2}{*}{\multicolumn{3}{|c|}{$\begin{array}{c}\text { Treatments }(\mathbf{T}) \\
\text { Interaction }(\mathbf{S} \times \mathbf{T})\end{array}$}} & & & & 0.005 & & & reatments & & & & & & & Treatments & & & & & \\
\hline & & & & & & 0.012 & & & eraction $(s$ & & & & & & & nteraction ( & & & & & \\
\hline
\end{tabular}


Table.2 Effect of different composite coatings on TSS ( ${ }^{0}$ Brix) and titratable acidity (\%) of guava cv. Sardar

\begin{tabular}{|c|c|c|c|c|c|c|c|c|c|c|c|c|c|c|c|c|c|c|c|c|c|}
\hline & \multicolumn{6}{|c|}{ TSS ( ${ }^{0}$ Brix) } & \multirow{4}{*}{ Mean } & \multicolumn{6}{|c|}{ TSS ( $\left({ }^{0}\right.$ Brix $)$} & \multirow{4}{*}{ Mean } & \multicolumn{6}{|c|}{ TSS ( ${ }^{0}$ Brix) } & \multirow{3}{*}{$\begin{array}{l}\text { Pooled } \\
\text { Mean }\end{array}$} \\
\hline Year & & & & & & & & & & & & & & & \multicolumn{6}{|c|}{ Pooled data } & \\
\hline \multirow[t]{2}{*}{ Treatments } & \multicolumn{6}{|c|}{ Storage intervals (days) } & & \multicolumn{6}{|c|}{ Storage intervals (days) } & & \multicolumn{6}{|c|}{ Storage intervals (days) } & \\
\hline & 0 & 3 & 6 & 9 & 12 & 15 & & 0 & 3 & 6 & 9 & 12 & 15 & & 0 & 3 & 6 & 9 & 12 & 15 & \\
\hline$T_{1}$ & 10.05 & 11.56 & 12.01 & 12.34 & 12.54 & 12.55 & 11.84 & 12.60 & 12.71 & 13.12 & 13.52 & 13.56 & 13.58 & 13.18 & 11.33 & 12.13 & 12.57 & 12.93 & 13.05 & 13.06 & 12.51 \\
\hline $\mathbf{T}_{2}$ & 10.05 & 11.44 & 12.04 & 12.33 & 12.55 & 12.57 & 11.83 & 12.60 & 12.77 & 13.11 & 13.55 & 13.61 & 13.62 & 13.21 & 11.33 & 12.11 & 12.58 & 12.94 & 13.08 & 13.10 & 12.52 \\
\hline $\mathbf{T}_{3}$ & 10.05 & 12.11 & 12.42 & 12.64 & 12.66 & 11.23 & 11.85 & 12.60 & 12.78 & 13.45 & 13.57 & 13.58 & 11.01 & 12.83 & 11.32 & 12.44 & 12.94 & 13.11 & 13.12 & 11.12 & 12.34 \\
\hline $\mathbf{T}_{4}$ & 10.05 & 12.03 & 12.34 & 12.66 & 12.67 & 11.52 & 11.88 & 12.60 & 12.81 & 13.55 & 13.64 & 13.66 & 10.06 & 12.72 & 11.32 & 12.42 & 12.95 & 13.15 & 13.17 & 10.79 & 12.30 \\
\hline $\mathbf{T}_{5}$ & 10.05 & 12.04 & 12.33 & 12.54 & 12.58 & 11.85 & 11.90 & 12.60 & 12.78 & 13.34 & 13.57 & 13.59 & 11.05 & 12.82 & 11.32 & 12.41 & 12.84 & 13.05 & 13.08 & 11.45 & 12.36 \\
\hline$T_{6}$ & 10.05 & 11.10 & 12.21 & 12.43 & 12.49 & 11.67 & 11.66 & 12.60 & 12.77 & 13.23 & 13.56 & 13.58 & 11.11 & 12.81 & 11.33 & 11.94 & 12.72 & 12.99 & 13.04 & 11.39 & 12.23 \\
\hline $\mathbf{T}_{7}$ & 10.05 & 12.43 & 12.71 & 12.98 & 8.11 & 6.35 & 10.44 & 12.60 & 13.01 & 13.89 & 14.01 & 9.04 & 6.28 & 11.47 & 11.32 & 12.72 & 13.30 & 13.49 & 8.57 & 6.32 & 10.96 \\
\hline Mean & 10.05 & 11.82 & 12.29 & 12.56 & 11.94 & 11.11 & & 12.60 & 12.80 & 13.38 & 13.63 & 12.95 & 10.96 & & 11.32 & 12.31 & 12.84 & 13.09 & 12.44 & 11.03 & \\
\hline \multicolumn{3}{|c|}{ Factors } & \multicolumn{2}{|c|}{$\mathrm{CD}$ at $5 \%$} & \multicolumn{3}{|c|}{$\mathrm{SE}(\mathrm{m})$} & \multicolumn{3}{|c|}{ Factors } & \multicolumn{2}{|c|}{$\mathrm{CD}$ at $5 \%$} & \multicolumn{2}{|c|}{$\mathrm{SE}(\mathrm{m})$} & \multicolumn{3}{|c|}{ Factors } & \multicolumn{2}{|c|}{$\mathrm{CD}$ at $5 \%$} & \multicolumn{2}{|c|}{$\mathrm{SE}(\mathrm{m})$} \\
\hline \multicolumn{3}{|c|}{ Storage Intervals (S) } & \multicolumn{2}{|c|}{0.137} & \multicolumn{3}{|c|}{0.049} & \multicolumn{3}{|c|}{ Storage Intervals (S) } & & & 0. & & & ge Interva & & & & & \\
\hline Treat & lents ( $T$ ) & & & & & 0.08 & & & eatments ( & & & & 0. & & & eatments & & & & & \\
\hline Intera & ion $(\mathbf{S} \times \mathbf{T}$ & & & & & 0.197 & & & raction $(\mathrm{S}$ & & & & 0. & & & raction (S & & & & & \\
\hline & & & Titrata & cidity ( & & & & & & Titratabl & idity $(\%)$ & & & & & & Titratabl & cidity $(\%)$ & & & \\
\hline Year & & & & & & & Mean & & & & & & & Mean & & & Poo & data & & & Pooled \\
\hline Treatments & & & Storage & vals $\left(d_{i}\right.$ & & & & & & Storage in & vals (day & & & & & & Storage in & vals (days & & & \\
\hline & 0 & 3 & 6 & 9 & 12 & 15 & & 0 & 3 & 6 & 9 & 12 & 15 & & 0 & 3 & 6 & 9 & 12 & 15 & \\
\hline$T_{1}$ & 0.25 & 0.25 & 0.21 & 0.19 & 0.17 & 0.16 & 0.21 & 0.32 & 0.31 & 0.29 & 0.26 & 0.23 & 0.18 & 0.27 & 0.29 & 0.28 & 0.25 & 0.22 & 0.20 & 0.17 & 0.24 \\
\hline $\mathbf{T}_{2}$ & 0.26 & 0.25 & 0.22 & 0.18 & 0.16 & 0.15 & 0.20 & 0.32 & 0.30 & 0.26 & 0.24 & 0.22 & 0.17 & 0.25 & 0.29 & 0.28 & 0.24 & 0.21 & 0.19 & 0.16 & 0.23 \\
\hline $\mathbf{T}_{3}$ & 0.26 & 0.24 & 0.20 & 0.16 & 0.14 & 0.13 & 0.19 & 0.32 & 0.31 & 0.23 & 0.21 & 0.16 & 0.14 & 0.23 & 0.29 & 0.28 & 0.21 & 0.19 & 0.15 & 0.14 & 0.21 \\
\hline $\mathbf{T}_{4}$ & 0.26 & 0.24 & 0.19 & 0.15 & 0.13 & 0.12 & 0.18 & 0.32 & 0.29 & 0.24 & 0.19 & 0.15 & 0.13 & 0.22 & 0.29 & 0.27 & 0.22 & 0.17 & 0.14 & 0.13 & 0.20 \\
\hline$T_{5}$ & 0.26 & 0.25 & 0.18 & 0.17 & 0.15 & 0.14 & 0.19 & 0.32 & 0.29 & 0.25 & 0.17 & 0.14 & 0.12 & 0.22 & 0.29 & 0.27 & 0.21 & 0.17 & 0.15 & 0.13 & 0.20 \\
\hline$T_{6}$ & 0.26 & 0.25 & 0.19 & 0.16 & 0.14 & 0.13 & 0.19 & 0.32 & 0.29 & 0.26 & 0.19 & 0.16 & 0.14 & 0.23 & 0.29 & 0.27 & 0.22 & 0.17 & 0.15 & 0.14 & 0.21 \\
\hline $\mathbf{T}_{7}$ & 0.26 & 0.20 & 0.13 & 0.11 & 0.11 & 0.09 & 0.15 & 0.32 & 0.23 & 0.19 & 0.13 & 0.11 & 0.10 & 0.18 & 0.29 & 0.21 & 0.16 & 0.12 & 0.11 & 0.09 & 0.16 \\
\hline Mean & 0.26 & 0.24 & 0.19 & 0.16 & 0.14 & 0.13 & & 0.32 & 0.29 & 0.25 & 0.20 & 0.17 & 0.14 & & 0.29 & 0.27 & 0.22 & 0.18 & 0.16 & 0.14 & \\
\hline
\end{tabular}

\begin{tabular}{|c|c|c|c|c|c|c|c|c|}
\hline Factors & $\mathrm{CD}$ at $5 \%$ & $\mathrm{SE}(\mathrm{m})$ & Factors & $\mathrm{CD}$ at $5 \%$ & $\mathrm{SE}(\mathrm{m})$ & Factors & $\mathrm{CD}$ at $5 \%$ & $\mathrm{SE}(\mathrm{m})$ \\
\hline Storage Intervals (S) & 0.003 & 0.001 & Storage Intervals (S) & 0.003 & 0.001 & Storage Intervals (S) & 0.002 & 0.001 \\
\hline Treatments (T) & 0.004 & 0.002 & Treatments ( $\mathrm{T}$ ) & 0.004 & 0.001 & Treatments $(\mathrm{T})$ & 0.004 & 0.001 \\
\hline Interaction $(\mathbf{S} \times \mathbf{T})$ & 0.011 & 0.004 & Interaction $(\mathrm{S} \times \mathrm{T})$ & 0.01 & 0.004 & Interaction $(\mathrm{S} \times \mathrm{T})$ & 0.01 & 0.003 \\
\hline
\end{tabular}


Table.3 Effect of different composite coatings on ascorbic acid (mg/100 g) and chlorophyll content (mg/g) of guava cv. Sardar

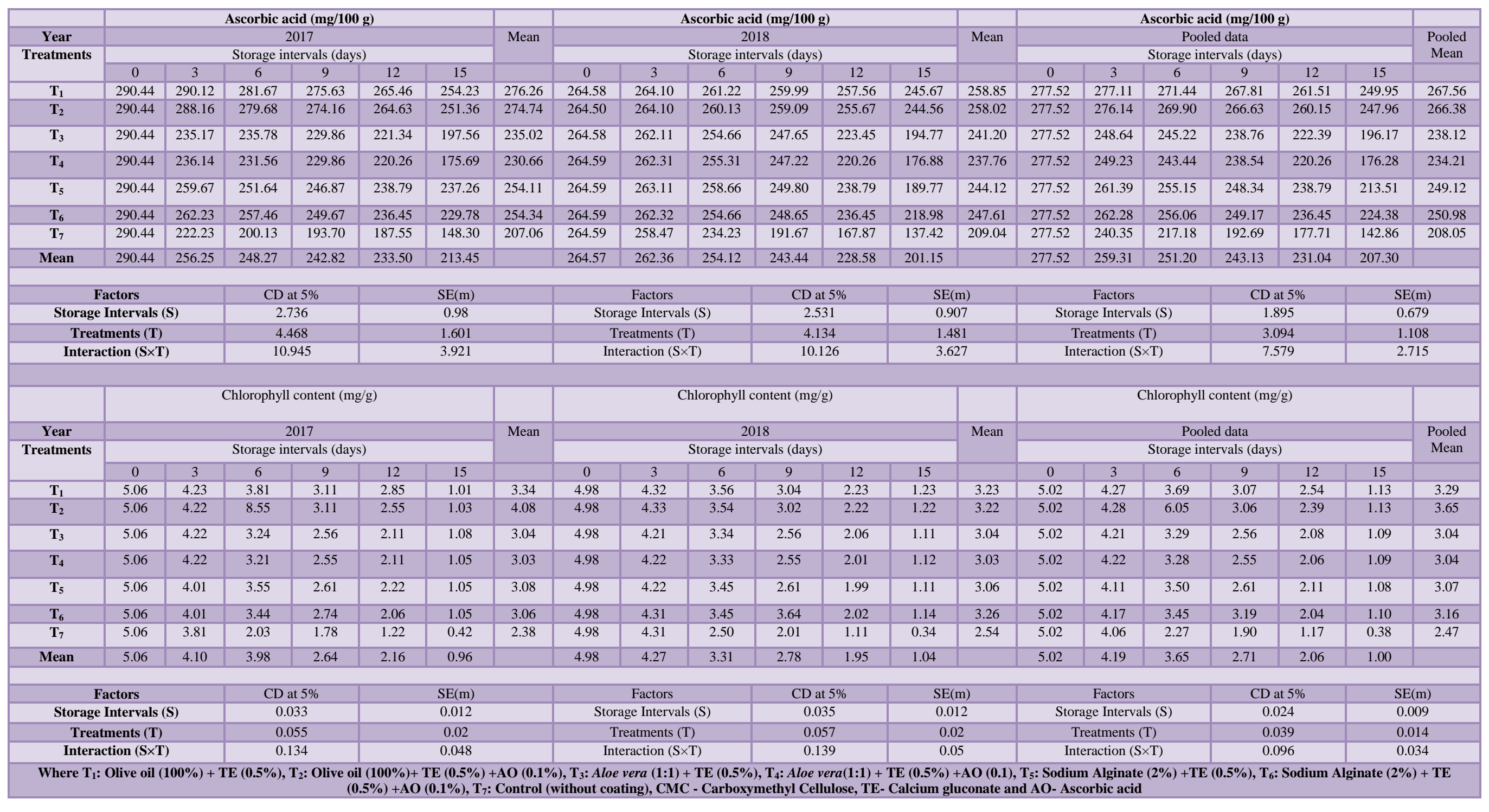


Maximum fruit titratable acidity $(0.21 \%)$ was recorded in $\left(\mathrm{T}_{1}\right)$ followed by $(0.20 \%)$ in $\mathrm{T}_{2}$ and minimum $(0.15 \%)$ was recorded in control $\left(\mathrm{T}_{7}\right)$ in winter season of 2017. During winter season 2018, maximum titratable acidity $(0.27 \%)$ was recorded in $\left(\mathrm{T}_{1}\right)$ and minimum $(0.18 \%)$ was recorded in control $\left(\mathrm{T}_{7}\right)$. In pooled data analysis maximum titratable acidity $(0.24 \%)$ was recorded in $\mathrm{T}_{1}$ i.e. Olive oil $(100 \%)+\mathrm{TE}$ $(0.5 \%)$ and minimum $(0.16 \%)$ was recorded in control $\left(\mathrm{T}_{7}\right)$. Slower decline in acidity in treated fruits compared to control might be due to delayed senescence and lower respiration rate in the fruits. Similar observations were also reported by Iqbal et al., 2017 in sweet orange and Baraiya et al., 2014 in carambola who also observed that coated fruits showed less reduction in acidity content during storage period. The data related to the effect of composite coatings during storage period on ascorbic acid of guava fruits cv. Sardar are given in Table 3. Persual of the data (Table 3) showed that maximum fruit ascorbic acid $(276.26 \mathrm{mg} / 100 \mathrm{~g})$ was recorded in $\left(\mathrm{T}_{1}\right)$ and minimum $(207.06 \mathrm{mg} / 100)$ was found in control $\left(\mathrm{T}_{7}\right)$ in winter season of 2017. During winter season 2018 maximum ascorbic acid (258.85 $\mathrm{mg} / 100 \mathrm{~g})$ was recorded in $\left(\mathrm{T}_{1}\right)$ and minimum $(209.04 \mathrm{mg} / 100 \mathrm{~g})$ was recorded in control $\left(\mathrm{T}_{7}\right)$. Similarly, in pooled data maximum ascorbic acid $(267.56 \mathrm{mg} / 100 \mathrm{~g})$ was recorded in $\mathrm{T}_{1}$ (Olive oil $(100 \%)+$ TE $0.5 \%$ ) followed by $(266.38 \mathrm{mg} / 100 \mathrm{~g})$ in $\mathrm{T}_{2}$, and minimum (208.05 $\mathrm{mg} / 100 \mathrm{~g})$ was recorded in control $\left(\mathrm{T}_{7}\right)$. Similarly, Iqbal et al., 2017 in sweet orange and Kumar et al., 2017 in guava were also reported that edible coating coated fruits retained higher ascorbic acid content during storage conditions. Kamboj and Kaur, 2018 observed that olive oil coated guava fruits cv. Allahabad Safeda had higher level of ascorbic acid content as compared to uncoated fruits during cold storage period. Further, olive oil coating was found more effective in the retention of ascorbic acid content because of the lowest gas permeability that inhibited the respiration rate. Maximum fruit chlorophyll content $(4.08 \mathrm{mg} / \mathrm{g})$ was found in $\mathrm{T}_{2}$ (Olive oil $100 \%+\mathrm{TE} 0.5 \%+\mathrm{AO} 0.1 \%$ ) and minimum chlorophyll content $(2.38 \mathrm{mg} / \mathrm{g})$ was retained in control $\left(\mathrm{T}_{7}\right)$ in winter season of 2017 (Table 3). Chlorophyll content during winter season 2018 was observed maximum $\left(3.23 \mathrm{mg} / \mathrm{g}\right.$ ) in $\mathrm{T}_{1}$ (Olive oil $100 \%+\mathrm{TE} 0.5 \%$ ) and minimum $(2.54 \mathrm{mg} / \mathrm{g})$ was observed in control $\left(\mathrm{T}_{7}\right)$. Similarly, in pooled data (Table 3 ), maximum chlorophyll content $(3.65 \mathrm{mg} / \mathrm{g})$ was recorded in $\mathrm{T}_{2}$ (Olive oil $100 \%+\mathrm{TE} 0.5 \%+\mathrm{AO}$ $0.1 \%)$ and minimum $(2.47 \mathrm{mg} / \mathrm{g})$ was noticed in control $\left(\mathrm{T}_{7}\right)$. Storage intervals affected chlorophyll content of fruit significantly which decreased gradually irrespective of the treatment as the storage period progressed. The change in colour of fruits from green to yellow continued over the storage period. Loss of chlorophyll is mediated through several processes involving the action of the enzyme chlorophyllase and photodegradation (Hussain et al., 2010). The green colour retention by application of coatings was reported by Shahid and Abbasi (2011) in sweet orange.

On the basis of findings, it may be concluded that olive oil + calcium guconate $0.1 \%$ and olive oil + calcium guconate $0.1 \%+$ ascorbic acid $0.1 \%$ were found quite effective in extending the shelf life as they retained greater physico-chemical properties like firmness, ascorbic acid, chlorophyll content with minimum physiological loss in weight during storage days.

\section{References}

Baraiya, N.S., Rao, T.V.R. and Thakkar, V.R. 2014. Enhancement of storability and quality maintenance of carambola (Averrhoa carambola L.) fruit by using composite edible coating. Fruit. 69: 195205.

Ergun, M. and Satici, F. 2012. Use of Aloe vera gel as biopreservative for 'Granny Smith' and 'Red Chief apples. The Journal of Animal and Plant Sciences. 22(2): 363368.

Gad, M.M. and Zagzog, O.A. 2017. Mixing xanthan gum and chitosan nano particles to form new coating for maintaining storage life and quality of Elmamoura 
guava fruits. Internatinal Journal of Current Microbiology and Applied Science. 6(11): 1582-1593.

Hussain, P.R., Meena, R.S., Dar, M.A. and Wani, A.M. 2010. Carboxymethyl cellulose coating and low-dose gamma irradiation improves storage quality and shelf life of pear (Pyrus communis L., cv. Bartlett/William). Journal of Food Science. 75: 586-596.

Iqbal, J., Rab, A., Sajid, M., Asim, S. and Bacha, S. 2017. Effect of partial coating of olive oil and storage duration on postharvest performance of sweet orange. Science International. 29(3):731-736.

Kamboj, P. and Kaur, A. 2018. Influence of Various Oil Coatings on the Shelf Life of Guava cv. Allahabad Safeda. Internatinal Journal of Pure and Applied Bioscience. 6 (3): 650-657.

Kessiane, S.M., Cristiane, F., Maria, C.M., Pamela, A. and Alcilene, R.M. 2012. Conservation of Williams's pear using edible coating with alginate and carrageenan. Ciencia e Tecnologia de Alimentos. 32, 679-684.

Kumar, A., Singh, O., Kohli, K. and Dubey, M.C. 2017. Effect of edible surface coatings on postharvest quality and shelf life of guava (Psidium guajava L. cv. Pant Prabhat) fruits. The Bioscan. 12(2): 825-832.

Moradinezhad, F., Naeimi, A. and Farhangfar, H. 2018. Influence of edible coatings on postharvest quality of fresh Chinese jujube fruits during refrigerated storage. Journal of Horticulture and Postharvest Research. 1(1): 1-14.

Nath, A., Deka, B.C., Singh, A., Patel, R.K., Paul, D. and Misra, L.K. 2012. Extension of shelf life of pear fruits using different packaging materials. Journal of Food Science and Technology. 49:556-563.

Nayek, S., Choudhury, I.H., Jaishee, N. and Roy, S. 2014. Spectrophotometric Analysis of chlorophylls and carotenoids from commonly grown fern species by using various extracting solvents. Research Journal of Chemical Sciences. 4(9): 63-69.

Othman, M.E., EL-Badry, N., Mahmoud, S. and Amer, M. 2017. The Effect of Edible Coating Contained Essential Oil on the Quality Attributes and Prolonging the Shelf Life of Guava Fruit. Middle East Journal of Agriculture Research. 6(1): 161-174.

Pal, R. K., Ahmad, M. H., Roy, S.K. and Singh, M. 2004. Influence of storage environment, surface coating and individual shrink rapping on quality assurance of guava (Psidium guajava) fruit. Plant foods for Human Nutrition. 59: 67-72.

Ranganna, S. 1986. Handbook of analysis and quality control for fruit and vegetable products. Tata Mcgraw Hill Publishing Co. Ltd., New Delhi. pp. 102-104.

Shahid, M.N. and Abbasi, N.A. 2011. Effect of bee wax coatings on physiological changes in fruits of sweet orange cv. 'Blood Red. Sarhad Journal of Agriculture. 27: 385- 394.

Zhu, X., Wang, Q.M., Cao, J.K. and Jiang, W.B. 2008. Effects of chitosan coating on postharvest quality of mango (Mangifera indica L.cv. Tainong) fruits. Journal of Food Process and Preservation. 32: 770784.

\section{How to cite this article:}

Karishma Kohli, Shailesh Tripathi, Ankit Kumar, Ajit Kumar, Omveer Singh, Rajesh Kumar and Satish Chand. 2019. Studies on Postharvest Changes in Physico-Biochemical Properties of Guava (Psidium guajava L.) cv. Sardar Influenced by Different Composite Coatings. Int.J.Curr.Microbiol.App.Sci. 8(08): 1417-1424. doi: https://doi.org/10.20546/ijcmas.2019.808.165 